\title{
Image Management and Communication in Patient Care: Perspectives on Implementation and Impact
}

\author{
Melvyn Greberman and Seong K. Mun
}

Editorial note: Dr Melvyn Greberman and Dr Seong K. Mun are the organizers of the First International Conference on Image Management and Communications in Patient Care (IMAC 89). They have provided the following comments on this subject. IMAC 89 will be held in Washington D.C. from June 4 to June 8, 1989. IMAC 91 will be held in Japan. For further information write to Dr Greberman.

Image management and communication (IMAC) systems are automated and integrated systems that capture digital medical images and related patient information and transmit them electronically, display them for interpretation, and store them for future retrieval. The IMAC system concept includes images and relevant information from all clinical sources. The First International Conference on Image Management and Communication in Patient Care (IMAC 89) provides a forum for expert presentations, poster sessions, and discussion and debate among all attendees interested in the implementation and impact of IMAC systems. Plenary sessions provide an international perspective and explore the role of image-based information in patient care, approaches to improved IMAC systems, current technical barriers, quality of care issues, evaluation approaches, and scenarios for the future. Invited participants are from North America, Europe, Japan, Australia, and the WHO. Conference organizers are working with numerous professional organizations and representatives of meetings which focus on IMAC-related technology to complement, and not duplicate, the contribution of other groups.

- 1989 by W.B. Saunders Company

KEY WORDS: Image management, picture archiving and communication systems, standards, technology assessment, evaluation.

$\mathbf{T}$ HE MANAGEMENT of the vast amounts of medical images and other information generated during the clinical care process is a growing problem. This problem is the result of several factors, including expensive technological advances, an increased emphasis on efficiency and cost-containment/-reduction, and changes in professional and organizational relationships and roles. Solutions to the problem will increasingly require the use of advanced computerbased technologies in data storage, image display, communication, and the application of human engineering principles.

The progress of individual technologies has been rapid in some areas, particularly in the digital imaging modalities used in radiology. The development and the widespread implementation and acceptance by users of systems that integrate the images from these modalities and from relevant clinical information has, however, been slow in coming. As experience accumulates in the clinical application of image management and communication (IMAC) systems, including teleradiology and picture archiving and communication systems (PACS), a critical assessment of their role in patient care and of their professional and economic impacts will be useful in developing future strategies for scientists, users, planners, regulators, and manufacturers.

Expansion of the IMAC system concept beyond radiology is becoming more important with the increased application of digital imaging technology in other clinical disciplines. Examples of digital imaging applications in other fields include radiation medicine (treatment planning and radiation delivery), gastroenterology (digital endoscopy), pathology (telepathology), orthopedics (design of prostheses), plastic/reconstructive surgery (treatment planning), neurosurgery (stereotaxy), cardiology (coronary angioplasty), ophthalmology (retinal angiography), dentistry (periodontitis therapy), and dermatology (skin cancer recognition). These applications are illustrative of the range of imaging applications for digital technologies; there is much variation in the degree to which such applications have been studied, implemented, and clinically accepted. In addition, medical educators and medical information organizations are planning to exploit advanced digital imaging and communication

From the Division of Technical Development, Office of Training and Assistance, Center for Devices and Radiological Health, Rockville, MD and the Division of Imaging Physics, Department of Radiology, Georgetown University Hospital, Washington, DC.

Address reprint requests to Melvyn Greberman, MD, MPH, Division of Technical Development, Office of Training and Assistance, Center for Devices and Radiological Health, 1901 Chapman Ave (HFZ-240), Rockville, MD 20857.

(C) 1989 by W.B. Saunders Company 0897-1889/89/0201-0012\$03.00/0 
technologies to improve the quality of medical education and the dissemination of medical knowledge.

The expense of medical imaging technology and of the research and development efforts associated with it, particularly in view of the cost-consciousness related to health care throughout the world, make it extremely important to learn from the related experiences of others; whether or not they are in the same or another clinical, engineering, or scientific discipline. Perhaps an even more fundamental reason for multidisciplinary cooperation in the research, development, implementation, and evaluation of IMAC systems is that the timely integration of accurate information from many clinical sources is essential to quality patient care.

There has been a high level of information sharing among PACS researchers in radiology. In addition to professional meetings (SPIE Medical Imaging meetings, Computer Assisted Radiology [CAR] meetings, Computer Applications in Radiology [Radiology Information System Consortium and the American College of Radiology], Radiological Society of North America Annual Meeting, EuroPACS annual meeting, Japan PACS meetings, etc), much time, effort, and money have been spent by major PACS investigators in the United States, Canada, United Kingdom, Netherlands, Germany, Italy, Sweden, Belgium, France, and Japan, to visit other laboratories and operational facilities. There have been a limited number of collaborative international research projects; those involving the Dutch National PACS Project and the University of Arizona provide good examples.

In addition to the radiology-oriented professional meetings, most of which have focused on technological developments in radiology, there are other meetings which have included a variety of clinical applications. They include the MEDINFO (Medical Informatics) conferences, Symposium on Computer Applications in Medical Care, annual meeting of the American Association for Medical Systems and Informatics, and the DOCUMED European Conference on the Application of Imaging and Visual Documentation in Medicine. Although these meetings provide opportunities for sharing information across discipline lines, it has become increasingly clear that the time is right for a multidisciplinary forum in which experienced users, clinicians, administrators, researchers, planners, system developers, regulators, and future users can explore major issues affecting IMAC system implementation and impact. The First International Conference on Image Management and Communication in Patient Care (IMAC 89) is designed to provide this forum; it will be held June 4-8, 1989, in Washington, DC. The organizers of IMAC 89 are working with representatives of professional organizations and with active participants in other meetings to complement, rather than duplicate, the efforts of others.

The following IMAC 89 plenary session headings provide a framework for the discussion of major issues associated with the implementation and impact of IMAC systems: (1) Scope and Challenges of IMAC in Patient Care: International Perspective; (2) Role of Image Based Information in Patient Care: Problem Definition; (3) Approaches to Improved Systems: Current Efforts and Possible Solutions; (4) Technical Barriers to Network Development and Information Exchange; (5) Assuring the Quality of Care; (6) Evaluation of IMAC Technologies; and (7) Future: What Might Happen Next?

\section{SCOPE AND CHALLENGES OF IMAC IN PATIENT CARE: INTERNATIONAL PERSPECTIVES}

The style of medical practice; the social, economic, and political environments; and the methods of patient-care financing vary among countries are likely to influence the development, implementation, and acceptance of IMAC systems. Participants in major IMAC projects may include representatives of universities (both clinical and technical personnel), industry, and government. The role of each group varies from project to project. The diversity of companies involved in IMAC technologies in a country, and the unique position of some companies within a country, may be important factors. Clinical turf perceptions may also be important, and these vary internationally.

ROLE OF IMAGE-BASED INFORMATION IN PATIENT CARE: PROBLEM DEFINITION

Some existing problems in patient care that IMAC systems can and should address include: 
(1) The availability of images and/or interpretative reports when needed to influence the clinical care process. In radiology, the many competing demands for a film during critical periods of inpatient care may cause problems in many hospitals. Poor reporting and/or information systems add to the difficulties; (2) Unnecessary images; this may be related to image availability but may, in addition, be related to image quality and the need for additional clinical information; (3) Communication of images and related information between the image interpreter and the referring physician or between institutions such as a diagnostic imaging center and a tertiary medical care facility; and (4) The integration of clinical information, including images from different modalities within a discipline, or from different disciplines (eg, radiology and pathology).

\section{APPROACHES TO IMPROVED SYSTEMS: CURRENT EFFORTS AND POSSIBLE SOLUTIONS}

Radiology has taken the leadership role in developing IMAC systems for patient care. Projects vary in scope from limited research activities concerned with specific components to extensive IMAC system efforts involving multiple radiology services and other clinical areas. The EuroPACS organization provides a European focus for researchers, clinicians, and others interested in PACS; its newsletter provides an extensive listing of individuals and PACS projects in Europe, North America, and Japan.

Teleradiology has had varying clinical acceptance but appears to meet a real need in certain parts of the United States and Canada. Telepathology is the principal area of IMAC system activity in pathology where the emphasis is on the remote transmission of images. The relative roles of digital and analog images are still being considered in pathology, whereas digital images are the standard for IMAC systems in radiology. Most other current applications of IMACrelated technology tend to focus on specific clinical applications. The Radiology. Information System Consortium, the American College of Radiology's Radiological Systems and Informatics Committee, and the United States National Library of Medicine are exploring the use of digital imaging technologies in medical education.
Much work is being done regarding the integration of medical information both in large computer-based systems and in small patientcarried components that are part of electronic medical record systems. Medical images are increasingly included among the data considered in both large and small systems. Communication standards activities are discussed later.

\section{TECHNICAL BARRIERS TO NETWORK DEVELOPMENT AND INFORMATION EXCHANGE}

Important issues remain in the areas of system integration, workstation design, data storage and database management, and communications and network configuration. The ACR-NEMA Digital Imaging and Communication Standard represents an important contribution to the field and a draft for Version 2.0 of the standard is being circulated. Discussions involving ACR-NEMA representatives with proponents of the Standard Product Interconnect (SPI) and with representatives of PACS standards activities in Japan are underway.

\section{ASSURING THE QUALITY OF CARE}

The management of image-based data electronically creates new requirements in patient care quality assurance. There are unanswered questions regarding the steps that need to be taken to insure clinical image quality and failsafe systems for storage, retrieval, and presentation. It is clear that answers to these questions will require input from many sources, including users, researchers, industry, and government.

\section{EVALUATION OF IMAC TECHNOLOGIES}

Although there is general agreement that assessment of IMAC system effectiveness is important, there is no general agreement as to how this should be done. As one gets further from localized department operations and deals with broader systems issues, technology assessment concepts are more difficult to apply, but may be more useful to society as a whole.

\section{FUTURE: WHAT MIGHT HAPPEN NEXT}

Predicting the future is always risky but some generalizations can be made: (1) the trend toward increased clinical applications of digital imaging technology will likely continue; (2) the degree to which this trend encourages interest in 
IMAC systems in radiology will depend on the extent to which film-based radiography is replaced by digital radiography; (3) user acceptance is important - this includes issues of userfriendly systems and of perceived clinical value in terms of efficiency and patient outcome; and (4) system integration will continue to be an important issue; consequently standards activities will continue to grow in importance and visibility. Efforts of umbrella groups such as the Healthcare Information Standards Coordinating Committee are needed if successful integration of images and information from a variety of clinical sources is to be a reality. 